Revised version 2

\title{
On the stochastic mechanics of the free relativistic particle
}

\author{
Michele Pavon \\ Dipartimento di Elettronica e Informatica \\ Università di Padova \\ and LADSEB-CNR, Italy \\ pavon@dei.unipd.it
}

November 6, 2018 


\begin{abstract}
Given a positive energy solution of the Klein-Gordon equation, the motion of the free, spinless, relativistic particle is described in a fixed Lorentz frame by a Markov diffusion process with non-constant diffusion coefficient. Proper time is an increasing stochastic process and we derive a probabilistic generalization of the equation $(d \tau)^{2}=$ $-\frac{1}{c^{2}} d X_{\nu} d X_{\nu} . \quad$ A random time-change transformation provides the bridge between the $t$ and the $\tau$ domain. In the $\tau$ domain, we obtain an $\mathbb{M}^{4}$-valued Markov process with singular and constant diffusion coefficient. The square modulus of the Klein-Gordon solution is an invariant, non integrable density for this Markov process. It satisfies a relativistically covariant continuity equation.
\end{abstract}

PACS number: $03.65 . \mathrm{Bz}$

Running title: Free relativistic particle 


\section{Introduction}

The purpose of this paper is to discuss a possible scenario for the free, spinless, relativistic particle, extending on Nelson's stochastic mechanics. This challenging problem has generated considerable interest in the past twenty years, see [3, 14, 5, 6, 10, 14, 18, 19, 25, 28, and references therein. As is wellknown, the main difficulty in this generalization stems from the non existence of nontrivial Markov diffusion processes possessing the required relativistic covariance, [7, 12]. A number of attempts have been made to circumvent this problem, for instance by considering different classes of stochastic processes, see e.g. [4, 5, 10, 28].

In this paper we propose a new approach. Corresponding to a positive energy solution of the Klein-Gordon equation, motion of the particle is described in a fixed Lorentz frame by an $\mathbb{R}^{3}$-valued Markov diffusion process with non-constant diffusion coefficient (local covariance matrix). The role of proper time is played here by an increasing stochastic process, namely a quadratic variation process. We also derive a natural probabilistic generalization of the equation $(d \tau)^{2}=-\frac{1}{c^{2}} d X_{\nu} d X_{\nu}$. A random time-change transformation provides the bridge between the $t$ and the $\tau$ domain. In the $\tau$ domain, we obtain an $\mathbb{M}^{4}$-valued Markov process with singular and constant diffusion coefficient. The fourth component is proportional to a stopping time. The square modulus of the Klein-Gordon solution is an invariant, non integrable density for this Markov process. It satisfies a relativistically covariant continuity equation. 


\section{Background on diffusion processes}

In this section, we review some essential concepts and results of the kinematics of stochastic mechanics. We refer to [22]- [24], [9] for a thorough account. In order to avoid any confusion, stochastic processes will be denoted by capital letters, as it is customary in probability. Let $(\Omega, \mathcal{E}, \mathbf{P})$ be a probability space. A stochastic process $\left\{X(t) ; t_{0} \leq t \leq t_{1}\right\}$ mapping $\left[t_{0}, t_{1}\right]$ into $L_{n}^{2}(\Omega, \mathcal{E}, \mathbf{P})$ is called a finite-energy diffusion with constant diffusion coefficient $I_{n} \sigma^{2}$ if the path $X(\omega)$ belongs a.s. to $C\left(\left[t_{0}, t_{1}\right] ; \mathbb{R}^{n}\right)$ (n-dimensional continuous functions) and

$$
X(t)-X(s)=\int_{s}^{t} \beta(\tau) d \tau+\sigma\left[W_{+}(t)-W_{+}(s)\right], \quad t_{0} \leq s<t \leq t_{1},
$$

where the forward drift $\beta(t)$ is at each time $t$ a measurable function of the past $\{X(\tau) ; 0 \leq \tau \leq t\}$, and $W_{+}(\cdot)$ is a standard, n-dimensional Wiener process with the property that $W_{+}(t)-W_{+}(s)$ is independent of $\{X(\tau) ; 0 \leq \tau \leq s\}$. Moreover, $\beta$ must satisfy the finite-energy condition

$$
E\left\{\int_{t_{0}}^{t_{1}} \beta(t) \cdot \beta(t) d t\right\}<\infty
$$

In [8], Föllmer has shown that a finite-energy diffusion also admits a reverse-

time differential. Namely, there exists a measurable function $\gamma(t)$ of the future $\left\{X(\tau) ; t \leq \tau \leq t_{1}\right\}$ called backward drift, and another Wiener process $W_{-}$such that

$$
X(t)-X(s)=\int_{s}^{t} \gamma(\tau) d \tau+\sigma\left[W_{-}(t)-W_{-}(s)\right], \quad t_{0} \leq s<t \leq t_{1}
$$


Moreover, $\gamma$ satisfies

$$
E\left\{\int_{t_{0}}^{t_{1}} \gamma(t) \cdot \gamma(t) d t\right\}<\infty,
$$

and $W_{-}(t)-W_{-}(s)$ is independent of $\left\{X(\tau) ; t \leq \tau \leq t_{1}\right\}$. Let us agree that $d t$ always indicates a strictly positive variable. For any function $f$ defined on $\left[t_{0}, t_{1}\right]$, let

$$
d_{+} f(t):=f(t+d t)-f(t)
$$

be the forward increment at time $t$, and

$$
d_{-} f(t)=f(t)-f(t-d t)
$$

be the backward increment at time $t$. For a finite-energy diffusion, Föllmer has also shown in [8] that the forward and backward drifts may be obtained as Nelson's conditional derivatives, namely

$$
\beta(t)=\lim _{d t \backslash 0} E\left\{\frac{d_{+} X(t)}{d t} \mid X(\tau), t_{0} \leq \tau \leq t\right\},
$$

and

$$
\gamma(t)=\lim _{d t \searrow 0} E\left\{\frac{d_{-} X(t)}{d t} \mid X(\tau), t \leq \tau \leq t_{1}\right\},
$$

the limits being taken in $L_{n}^{2}(\Omega, \mathcal{B}, P)$. It was finally shown in $[\mathbb{8}]$ that the one-time probability density $\rho(\cdot, t)$ of $X(t)$ (which exists for every $t>t_{0}$ ) is absolutely continuous on $\mathbb{R}^{n}$ and the following relation holds $\forall t>0$

$$
E\{\beta(t)-\gamma(t) \mid X(t)\}=\sigma^{2} \nabla \log \rho(X(t), t) .
$$

Let us introduce the current drift $v(t):=(\beta(t)+\gamma(t)) / 2$ and the osmotic drift $u(t):=(\beta(t)-\gamma(t)) / 2$. Notice that, when $\sigma$ tends to zero, $v$ tends to 
$\dot{X}$, and $u$ tends to zero. The finite-energy diffusion $X(\cdot)$ is called Markovian if there exist two measurable functions $b_{+}(\cdot, \cdot)$ and $b_{-}(\cdot, \cdot)$ such that $\beta(t)=$ $b_{+}(X(t), t)$ a.s. and $\gamma(t)=b_{-}(X(t), t)$ a.s., for all $t$ in $\left[t_{0}, t_{1}\right]$. The duality relation (2.7) now reads

$$
b_{+}(X(t), t)-b_{-}(X(t), t)=\sigma^{2} \nabla \log \rho(X(t), t) .
$$

This immediately gives the osmotic equation

$$
u(x, t)=\frac{\sigma^{2}}{2} \nabla \log \rho(x, t),
$$

where $u(x, t):=\left(b_{+}(x, t)-b_{-}(x, t)\right) / 2$. The probability density $\rho(\cdot, \cdot)$ of $X(t)$ satisfies (at least weakly) the Fokker-Planck equation

$$
\frac{\partial \rho}{\partial t}+\nabla \cdot\left(b_{+} \rho\right)=\frac{\sigma^{2}}{2} \Delta \rho .
$$

The latter can also be rewritten, in view of (2.8), as the equation of continuity of hydrodynamics

$$
\frac{\partial \rho}{\partial t}+\nabla \cdot(v \rho)=0
$$

where $v(x, t):=\left(b_{+}(x, t)+b_{-}(x, t)\right) / 2$.

Nelson's stochastic mechanics [22, 9, 23, [1] is a quantization procedure for classical dynamical systems based on diffusion processes. Given a quantum evolution $\left\{\psi(x, t) ; t_{0} \leq t \leq t_{1}\right\}$, namely a solution of the Schrödinger equation

$$
\frac{\partial \psi}{\partial t}=\frac{i \hbar}{2 m} \Delta \psi-\frac{i}{\hbar} V(x) \psi,
$$

satisfying Carlen's finite action condition

$$
\|\nabla \psi\|_{2}^{2} \in L_{\mathrm{loc}}^{1}\left[\left[t_{0},+\infty\right)\right]
$$


it is possible to construct a measure on path-space under which the canonical coordinate process $X$ is a finite-energy Markov diffusion process. Writing $\psi(x, t)=\exp R(x, t)+\frac{i}{\hbar} S(x, t)$, we have that the process $X$ has current and osmotic drift fields given, respectively, by $v(x, t)=\frac{1}{m} \nabla S(x, t)$, and $u(x, t)=$ $\frac{\hbar}{m} \nabla R(x, t)$. In particular, the (forward) Ito differential of $X$ is given by

$$
\begin{aligned}
d X(t) & =\left[\frac{\hbar}{m} \nabla(\Re \log \psi(X(t), t)+\Im \log \psi(X(t), t))\right] d t \\
& +\sqrt{\frac{\hbar}{m}} d W_{+}(t),
\end{aligned}
$$

see [2], [1, Chapter IV], and references therein. Moreover, the probability density $\rho(\cdot, t)$ of $x(t)$ satisfies

$$
\rho(x, t)=|\psi(x, t)|^{2}, \quad \forall t \in\left[t_{0}, t_{1}\right] .
$$

We now need to recall the generalization to Markov processes with diffusion coefficient of the form $I_{n} \sigma(x, t)^{2}$, with $\sigma(x, t) \in \mathbb{R}$, the above kinematics, see e.g. [21], [20], [17] and [13]. Let $\left\{X(t) ; t_{0} \leq t \leq t_{1}\right\}$ be an $n$-dimensional Markov diffusion process whose increments admit the two representations

$$
\begin{aligned}
& d_{+} X(t)=b_{+}(X(t), t) d t+\sigma_{+}(X(t), t) d_{+} W_{+}(t), \\
& d_{-} X(t)=b_{-}(X(t), t) d t+\sigma_{-}(X(t), t) d_{-} W_{-}(t),
\end{aligned}
$$

where the drifts and diffusion coefficients are sufficiently regular. Here, $W_{+}$ and $W_{-}$are standard, n-dimensional Wiener processes adapted to the past and the future, respectively, of the process $X$. Then, the following relations hold

$$
\begin{array}{r}
\sigma_{+}(x, t)=\sigma_{-}(x, t)=\sigma(x, t) \\
b_{+}(x, t)-b_{-}(x, t)=\frac{1}{\rho} \nabla\left(\sigma(x, t)^{2} \rho(x, t)\right) .
\end{array}
$$


Letting, as usual, $v:=\left(b_{+}+b_{-}\right) / 2$ denote the current drift, we have, as before, that the Fokker-Planck equation can be rewritten as the continuity equation (2.10). Let $R(x, t):=\frac{1}{2} \log \left(\sigma(x, t)^{2} \rho(x, t)\right)$. Then, observing that (2.18) can be rewritten as

$$
b_{+}(x, t)-b_{-}(x, t)=\sigma(x, t)^{2} \nabla \log \left(\sigma(x, t)^{2} \rho(x, t)\right),
$$

we get the following expression for the osmotic drift $u$

$$
u(x, t)=\sigma(x, t)^{2} \nabla R(x, t) .
$$

\section{Description in a fixed inertial frame}

For the definitions and results about continuous martingales that occur in this section, we refer the reader to the Appendix and, for a thorough treatment, to [15] and [16]. Let $\varphi:=\exp \left[R+\frac{i}{\hbar} S\right]$ be a solution of the Klein-Gordon equation

$$
\Delta \varphi-\frac{1}{c^{2}} \frac{\partial^{2}}{\partial t^{2}} \varphi=\frac{m^{2} c^{2}}{\hbar^{2}} \varphi
$$

on $[0, \infty)$, such that $S$ satisfies on the same time interval

$$
\frac{\partial S}{\partial t}+c \sqrt{m^{2} c^{2}+\nabla S \cdot \nabla S}=0 .
$$

This is precisely the family of solutions that was considered in [19]. Indeed, let

$$
\begin{array}{r}
\rho(x, t)=\frac{i \hbar}{2 m c^{2}}\left(\bar{\varphi} \frac{\partial \varphi}{\partial t}-\varphi \frac{\partial \bar{\varphi}}{\partial t}\right)(x, t)=|\varphi(x, t)|^{2} \times\left(-\frac{1}{m c^{2}} \frac{\partial S}{\partial t}(x, t)\right), \\
\mathbf{j}(x, t)=\frac{\hbar}{2 m i}(\bar{\varphi} \nabla \varphi-\varphi \nabla \bar{\varphi})(x, t)=|\varphi(x, t)|^{2} \times\left(\frac{1}{m} \nabla S(x, t)\right) .
\end{array}
$$


First of all, observe that because of (3.1), the pair $(\rho, \mathbf{j})$ satisfies a continuity type equation

$$
\frac{\partial \rho}{\partial t}+\nabla \cdot \mathbf{j}=0
$$

Moreover, from (3.2) it also follows that

$$
\begin{array}{r}
\rho \geq 0 \\
\mathbf{j} \cdot \mathbf{j}-c^{2} \rho^{2}=-c^{2}|\varphi|^{4} .
\end{array}
$$

The latter two conditions are equivalent to (5.10) in [19, p.4705]. This class of solutions is non empty. It contains at least all positive-frequency plane-wave solutions, namely solutions of the form

$$
\varphi(x, t)=\exp \left[(i / \hbar)\left(\mathbf{p} \cdot x-p^{0} t\right)\right]
$$

where

$$
p^{0}=+c \sqrt{\mathbf{p} \cdot \mathbf{p}+m^{2} c^{2}} .
$$

Let

$$
\rho_{0}(x):=\frac{i \hbar}{2 m c^{2}}\left(\bar{\varphi} \frac{\partial \varphi}{\partial t}-\varphi \frac{\partial \bar{\varphi}}{\partial t}\right)(x, 0)=|\varphi(x, 0)|^{2} \times\left(-\frac{1}{m c^{2}} \frac{\partial S}{\partial t}(x, 0)\right) .
$$

It follows from (3.2) that $\rho_{0}(x) \geq 0$. Let us suppose that $\rho_{0}$ integrates to one. Suppose that $\{X(t) ; t \geq 0\}$ is a Markov diffusion process such that $X(0)$ is distributed according to $\rho_{0}$, and having forward differential

$$
d_{+} X(t)=\left(\frac{\frac{1}{m} \nabla S+\frac{\hbar}{m} \nabla R}{-\frac{1}{m c^{2}} \frac{\partial S}{\partial t}}\right)(X(t), t) d t+\sqrt{\frac{\hbar}{m}} \frac{1}{\sqrt{-\frac{1}{m c^{2}} \frac{\partial S}{\partial t}(X(t), t)}} d_{+} W_{+}(t),
$$


where $W_{+}(\cdot)$ is a standard, 3-dimensional Wiener process. In the previous notation, we have

$$
\sigma^{2}(x, t):=\frac{\hbar}{-\frac{1}{c^{2}} \frac{\partial S}{\partial t}(x, t)} .
$$

A standard calculation [27] shows that $\rho(x, t)$ in (3.3) satisfies the FokkerPlanck equation corresponding to (3.7). Hence, $\rho(x, t)$ is the probability density of $X(t)$. Moreover, the current drift of $X$ is

$$
v(X(t), t):=\left(\frac{\frac{1}{m} \nabla S}{-\frac{1}{m c^{2}} \frac{\partial S}{\partial t}}\right)(X(t), t),
$$

and the osmotic drift is given by

$$
u(X(t), t)=\left(\frac{\frac{\hbar}{m} \nabla R}{-\frac{1}{m c^{2}} \frac{\partial S}{\partial t}}\right)(X(t), t) .
$$

Let us define

$$
M^{i}(t):=\int_{0}^{t} \frac{1}{\sqrt{-\frac{1}{m c^{2}} \frac{\partial S}{\partial s}(X(s), s)}} d_{+} W_{+}^{i}(s), \quad t \geq 0 \quad i=1,2,3 .
$$

The stochastic processes $\left\{M^{i}(t) ; t \geq 0\right\}$ are continuous local martingales with the same quadratic variation

$$
<M>(t)=<M^{i}>(t):=\int_{0}^{t} \frac{1}{-\frac{1}{m c^{2}} \frac{\partial S}{\partial s}(X(s), s)} d s .
$$

Moreover, their cross variations satisfy

$$
<M^{i}, M^{j}>(t)=0, \quad 1 \leq i \neq j \leq 3, \quad \forall t \geq 0 .
$$

Observe that $<M>(t)$ is a strictly increasing process with differentiable sample paths. It satisfies, in view of (3.2) and (3.8),

$$
d<M>(t)=\frac{1}{-\frac{1}{m c^{2}} \frac{\partial S}{\partial t}(X(t), t)} d t
$$




$$
\begin{aligned}
& =\sqrt{\frac{m^{2} c^{2}}{m^{2} c^{2}+\nabla S(X(t), t) \cdot \nabla S(X(t), t)}} d t \\
& =\sqrt{1-\frac{\nabla S(X(t), t) \cdot \nabla S(X(t), t)}{m^{2} c^{2}+\nabla S(X(t), t) \cdot \nabla S(X(t), t)}} d t \\
& =\sqrt{1-\frac{v(X(t), t) \cdot v(X(t), t)}{c^{2}}} d t .
\end{aligned}
$$

If for some $t$ and $\omega$ we have $v(X(t, \omega), t)=0$, then $d<M>(t)=d t$. We can therefore think of $d<M>(t)$ as of an increment of the particle random proper time. Notice that (3.13) implies

$$
\frac{d<M>(t)}{d t} \leq 1, \quad \text { a.s. }
$$

Hence,

$$
<M>(t) \leq t, \quad \text { a.s. }, \quad \forall t \geq 0 .
$$

Furthermore, we have the following property of $d<M>(t)$. Let us first recall that, in view of (2.5) and (2.6), we have

$$
v(X(t), t)=\lim _{d t \searrow 0} E\left\{\frac{d_{s} X(t)}{d t} \mid X(t)\right\},
$$

where the symmetric increment of $X$ at time $t$ is defined by

$$
d_{s} X(t):=\frac{d_{+} X(t)+d_{-} X(t)}{2}=\frac{X(t+d t)-X(t-d t)}{2} .
$$

We can therefore rewrite (3.13) as follows.

$$
\begin{array}{r}
c^{2}[d<M>(t)]^{2}=-\left[(i c)^{2}+v(X(t), t) \cdot v(X(t), t)\right](d t)^{2} \\
=-\left[\left(\frac{d(i c t)}{d t}\right)^{2}+v(X(t), t) \cdot v(X(t), t)\right](d t)^{2} \\
=-\left[(d(i c t))^{2}+E\left\{d_{s} X(t) \mid X(t)\right\} \cdot E\left\{d_{s} X(t) \mid X(t)\right\}+o\left((d t)^{2}\right)\right] .
\end{array}
$$


Let us introduce

$$
X_{\nu}(t)=\left(\begin{array}{c}
X(t) \\
i c t
\end{array}\right) .
$$

Then (3.16) can be written as follows

$$
[d<M>(t)]^{2}=-\frac{1}{c^{2}} E\left\{d_{s} X_{\nu}(t) \mid X_{\nu}(t)\right\} \cdot E\left\{d_{s} X_{\nu}(t) \mid X_{\nu}(t)\right\}+o\left((d t)^{2}\right),
$$

generalizing the relation $(d \tau)^{2}=-\frac{1}{c^{2}} d X_{\nu} d X_{\nu}$ of classical relativistic mechanics.

\section{From the $t$ to the $\tau$ formulation: A random time-change}

An important consequence of Levy's characterization of the Wiener process,

see e. g. [16, p. 82], is that any continuous local martingale may be viewed as a time-changed Wiener process, cf. Theorem A.2 in the Appendix. For $\tau \geq 0$, let us introduce the stopping time

$$
T(\tau):=\inf \{\sigma \geq 0:<M>(\sigma) \geq \tau\}
$$

(In the case when the probability of the event $\left\{\omega: \lim _{t \rightarrow \infty}<M>(t, \omega)=\right.$ $\infty\}$ is strictly less than one, the stopping time $T(\tau)$ has to be suitably modified, see [15, pp.174-175]). Notice that

$$
<M>(T(\tau))=\tau, \quad T(<M>(t))=t
$$

Then, the processes

$$
\tilde{W}_{+}^{i}(\tau):=M_{T(\tau)}^{i}, \quad i=1,2,3,
$$


are, standard, one-dimensional Wiener processes. Moreover, in view of property (3.12), we can apply a theorem of F. B. Knight (see Theorem A.3 in the Appendix), and conclude that the processes $\tilde{W}_{+}^{i}(\tau)$ are pairwise independent, and can, consequently, be viewed as the components of a standard, three-dimensional Wiener process $\tilde{W}_{+}(\tau)$. Let us introduce the stochastic process

$$
\tilde{X}(\tau):=X(T(\tau)), \quad \tau \geq 0 .
$$

In view of (3.7), (3.11), and (4.1), the forward differential of $\tilde{X}$ is given by

$$
d_{+} \tilde{X}(\tau)=\left(\frac{1}{m} \nabla S+\frac{\hbar}{m} \nabla R\right)(\tilde{X}(\tau), T(\tau)) d \tau+\sqrt{\frac{\hbar}{m}} d_{+} \tilde{W}_{+}(\tau) .
$$

Notice that $\tilde{X}(\tau)$ is a non-Markovian $\mathbb{R}^{3}$-valued diffusion process with constant diffusion coefficient $\frac{\hbar}{m} I_{3}$. The Markov property has therefore been destroyed by the random time change. From the first relation in (4.1), we get

$$
\frac{d T(\tau)}{d \tau}=\left[\frac{d<M>}{d t}(T(\tau))\right]^{-1} .
$$

In view of (3.11), we then get

$$
\frac{d T(\tau)}{d \tau}=-\frac{1}{m c^{2}} \frac{\partial S}{\partial t}(\tilde{X}(\tau), T(\tau)) .
$$

Let us now define $\tilde{X}^{4}(\tau):=i c T(\tau), \tilde{S}\left(x_{\nu}\right)=\tilde{S}\left(x, x^{4}\right):=S(x, t)$, and $\tilde{R}\left(x_{\nu}\right)=$ $\tilde{R}\left(x, x^{4}\right):=R(x, t)$. Equations (4.2) and (4.3) can now be rewritten as

$$
\begin{aligned}
d_{+} \tilde{X}(\tau) & =\left(\frac{1}{m} \nabla \tilde{S}+\frac{\hbar}{m} \nabla \tilde{R}\right)\left(\tilde{X}(\tau), \tilde{X}^{4}(\tau)\right) d \tau+\sqrt{\frac{\hbar}{m}} d_{+} \tilde{W}_{+}(\tau) \\
d_{+} \tilde{X}^{4}(\tau) & =\frac{1}{m} \frac{\partial \tilde{S}}{\partial x^{4}}\left(\tilde{X}(\tau), \tilde{X}^{4}(\tau)\right) d \tau
\end{aligned}
$$


Notice that $\tilde{X}_{\nu}(\tau)$ defined by

$$
\tilde{X}_{\nu}(\tau)=\left(\begin{array}{c}
\tilde{X}(\tau) \\
\tilde{X}_{4}(\tau)
\end{array}\right)
$$

is an $\mathbb{M}^{4}$-valued Markovian stochastic process with marginal density at $\tau=0$ given by $\rho_{0}(x) \cdot \delta\left(x^{4}\right)$ since $T(0)=0$ a.s. Let us introduce the diffusion matrix

$$
\Sigma^{2}:=\left(\begin{array}{cc}
\frac{\hbar}{m} I_{3} & 0 \\
0 & 0
\end{array}\right)
$$

. Then (4.4)-(4.5) can be rewritten in the form

$$
d_{+} \tilde{X}_{\nu}(\tau)=\left(\frac{1}{m} \nabla_{\nu} \tilde{S}+\Sigma^{2} \nabla_{\nu} \tilde{R}\right)\left(\tilde{X}_{\nu}(\tau)\right) d \tau+\Sigma d_{+} \tilde{W}_{\nu}(\tau)
$$

where $\tilde{W}_{\nu}$ is any standard, four-dimensional Wiener process whose first three components form $\tilde{W}_{+}$. The Fokker-Plank equation is then

$$
\frac{\partial \rho_{\nu}}{\partial \tau}+\nabla_{\nu} \cdot\left[\left(\frac{1}{m} \nabla_{\nu} \tilde{S}+\Sigma^{2} \nabla_{\nu} \tilde{R}\right) \rho_{\nu}\right]=\frac{1}{2} \Sigma^{2} \Delta_{\nu} \rho_{\nu} .
$$

Because of (3.1), $\varphi\left(x_{\nu}\right):=\exp \left[\tilde{R}\left(x_{\nu}\right)+\frac{i}{\hbar} \tilde{S}\left(x_{\nu}\right)\right]$ satisfies

$$
\nabla_{\nu} \cdot \nabla_{\nu} \varphi=\frac{m^{2} c^{2}}{\hbar^{2}} \varphi
$$

The latter is equivalent to the system of p.d.e.'s

$$
\begin{aligned}
-\frac{1}{2 m} \nabla_{\nu} \tilde{S} \cdot \nabla_{\nu} \tilde{S}+\frac{\hbar^{2}}{2 m}\left[\nabla_{\nu} \tilde{R} \cdot \nabla_{\nu} \tilde{R}+\nabla_{\nu} \cdot \nabla_{\nu} \tilde{R}\right] & =\frac{m c^{2}}{2} \\
\frac{1}{m} \nabla_{\nu} \tilde{S} \cdot \nabla_{\nu} \tilde{R}+\frac{1}{2 m} \nabla_{\nu} \cdot \nabla_{\nu} \tilde{S} & =0 .
\end{aligned}
$$

Let

$$
\tilde{\rho}\left(x_{\nu}\right)=\left|\varphi\left(x_{\nu}\right)\right|^{2} .
$$


From (4.9), we get that $\tilde{\rho}\left(x_{\nu}\right)$ satisfies (4.7). Thus, $\mu\left(d x_{\nu}\right):=\tilde{\rho}\left(x_{\nu}\right) d x_{\nu}$ is an invariant ( $\sigma$-finite) measure for (4.4)-(4.5). Notice that $\tilde{\rho}\left(x_{\nu}\right)$ also satisfies the manifestly covariant equation

$$
\nabla_{\nu} \cdot\left(\tilde{\rho} \frac{1}{m} \nabla_{\nu} \tilde{S}\right)=0 .
$$

If we give $\mu\left(d x_{\nu}\right):=\tilde{\rho}\left(x_{\nu}\right) d x_{\nu}$ as initial measure to (4.6), the probabilistic picture is lost. Nevertheless, it is possible to make sense of the time-reversed diffusion along the lines of [1, pp.44-45]. Moreover, consider the generator of $\tilde{X}_{\nu}$ acting on smooth functions with compact support

$$
\left(\frac{1}{m} \nabla_{\nu} \tilde{S}+\Sigma^{2} \nabla_{\nu} \tilde{R}\right) \cdot \nabla_{\nu}+\frac{1}{2} \Sigma^{2} \Delta_{\nu}
$$

The adjoint of this operator with respect to the measure $\mu\left(d x_{\nu}\right)$ [22, p.104] is given by

$$
\left(\frac{1}{m} \nabla_{\nu} \tilde{S}-\Sigma^{2} \nabla_{\nu} \tilde{R}\right) \cdot \nabla_{\nu}-\frac{1}{2} \Sigma^{2} \Delta_{\nu}
$$

Hence, in spite of the lack of a probabilistic picture we can still think of $\frac{1}{m} \nabla_{\nu} \tilde{S}$ as of a bilateral velocity field associated to the equilibrium measure $\mu\left(x_{\nu}\right)$. Then, recalling that (3.2) implies

$$
\nabla_{\nu} \tilde{S}\left(x_{\nu}\right) \cdot \nabla_{\nu} \tilde{S}\left(x_{\nu}\right)=-m^{2} c^{2}
$$

we get a $\tau$-domain counterpart of relation (3.17).

\section{Conclusion and outlook}

Although the process (4.4)-(4.5) is not relativistically covariant, our description does not appear to be in conflict with classical relativistic mechanics nor 
with Nelson's nonrelativistic theory. Indeed, in the case when we let the noise intensity in (3.7) tend to zero, we recover the equations of classical special relativity. In particular, the quadratic variation $<M>$ defined by (3.11) tends to proper time $\tau$ and $T(\tau)$ tends to $t(\tau)$. On the other hand, when $v \cdot v<<c^{2}$, motion of the particle in a fixed inertial frame is described by a Markov diffusion process with diffusion coefficient nearly equal to $\frac{\hbar}{m}$. The Schrödinger equation is then recovered through some appropriate procedure neglecting terms multiplied by $\hbar^{2} / c$, cf. [19, p. 4708].

Acknowledgments: The author wishes to thank Paolo Dai Pra for some helpful comments on the results of this paper, and Lorenza Viola for several useful conversations on relativistic stochastic mechanics.

\section{References}

[1] Ph. Blanchard, Ph. Combe and W. Zheng, Math. and Physical Aspects of Stochastic Mechanics, Lect. Notes in Physics vol. 281, Springer-Verlag, New York, 1987.

[2] E. Carlen, Conservative diffusions, Comm. Math. Phys. 94 (1984), 293315.

[3] E. Carlen, Ann. Inst. H. Poincaré A42 (1985), 407.

[4] G. F. De Angelis, Stochastic mechanics of a relativistic spinless particle, J. Math. Phys. 31 (1990), 1408.

[5] G.F. De Angelis and M. Serva, Ann. Inst. H. Poincaré 53 (1990), 301.

[6] D. Dohrn and F. Guerra, Phys. Rev. D. 31 (1985), 1408.

[7] R. M. Dudley, Ark. f. Mat. 6 (1965), 241.

[8] H. Föllmer, in: Stochastic Processes - Mathematics and Physics, Lect. Notes in Math. 1158 (Springer-Verlag, New York,1986), p. 119.

[9] F. Guerra, Structural aspects of stochastic mechanics and stochastic field theory, Phys.Rep. 77 (1981)263. 
[10] F. Guerra and P. Ruggero, Lett. Nuovo Cimento 23 (1978), 529.

[11] H. Goldstein, Classical Mechanics, Addison-Wesley Publ. Co.,Reading, Mass., Second Edition, 1980.

[12] R. Hakim, Relativistic stochastic processes, J. Math. Phys. 9 (1968), 1805.

[13] U.G.Haussmann and E.Pardoux, Time reversal of diffusions, The Annals of Probability 14, 1986, 1188.

[14] P.N.Kaloyerou and J.P. Vigier, J. Phys. A 22 (1989), 663.

[15] I. Karatzas and S. E. Shreve, Brownian Motion and Stochastic Calculus, Springer-Verlag, New York, 1988.

[16] R.Liptser and A.Shiryayev, Statistics of Random Processes I, SpringerVerlag,New York,1977.

[17] L. M. Morato, On the dynamics of diffusions and the related general electromagnetic potentials, J. Math. Phys. 23 (1982), 1020.

[18] L. M. Morato, A new approach to the problem of relativistic kinematics in stochastic mechanics and a derivation of Klein-Gordon equation, in Probabilistic Methods in Mathematical Physics, edited by F.Guerra, M.Loffredo and C.Marchioro Eds., World Scientific, 1992, pp. 318-328.

[19] L. M. Morato and L. Viola, Markov diffusions in comoving coordinates and stochastic quantization of the free relativistic spinless particle, $J$. Math. Phys. 36 (1995), 4691.

[20] M.Nagasawa, The adjoint process of a diffusion with reflecting barrier, Kodai Math.Sem.Rep. 13, 1961, 235.

[21] E. Nelson, The adjoint Markov process, Duke Math. J. 25 (1958), 671.

[22] E. Nelson,Dynamical Theories of Brownian Motion, Princeton University Press, Princeton, 1967.

[23] E. Nelson, Quantum Fluctuations, Princeton University Press, Princeton, 1985.

[24] E. Nelson, Stochastic mechanics and random fields, inĖcole d'Ètè de Probabilitès de Saint-Flour XV-XVII, edited by P. L. Hennequin, Lecture Notes in Mathematics, Springer-Verlag, New York, 1988, vol.1362, pp. $428-450$.

[25] E. Nelson, Clock Space, in Probabilistic Methods in Mathematical Physics, edited by F.Guerra, M.Loffredo and C.Marchioro Eds., World Scientific, 1992, pp. 329-333.

[26] H. Rund. The Hamilton-Jacobi Theory in the Calculus of Variations, Van Nostrand, London, 1966. 
[27] S. S. Schweber, An Introduction to Relativistic Quantum Field Theory, Harper \& Row and John Weatherhill, Tokyo, 1964.

[28] M. Serva, Relativistic stochastic processes associated to Klein-Gordon equation, Ann. Inst. H. Poincaré 49 (1988), 415.

\section{A Continuous Martingales}

We collect in this appendix a few basic facts about continuous martingales. We refer the reader to [15] and [16] for the proofs and more information. Let $(\Omega, \mathcal{A}, \mathbf{P})$ be a probability space, and let $\mathcal{F}:=\left(\mathcal{F}_{t}\right), t \in\left[t_{0}, t_{1}\right]$, be a non-decreasing family of sub $\sigma$-algebras of $\mathcal{A}$. It will be always assumed that $\mathcal{F}_{t_{0}}$ contains all the zero-probability sets in $\mathcal{A}$, and that the filtration is right-continuous, namely $\left\{\cap \mathcal{F}_{t}, t>s\right\}=\mathcal{F}_{s}$, for every $s$. A stochastic process $X:=\left\{X(t) ; t \in\left[t_{0}, t_{1}\right]\right\}$ is said to be continuous if it has continuous trajectories $X(t, \omega), t \in\left[t_{0}, t_{1}\right]$, with probability one. It is called $\mathcal{F}$-adapted if, for every $t$ in $\left[t_{0}, t_{1}\right], X(t)$ is $\mathcal{F}_{t}$-measurable. In that case, it is customary to write $X=\left(X(t), \mathcal{F}_{t}\right)_{t \in\left[t_{0}, t_{1}\right]}$. The stochastic process $M=\left(M(t), \mathcal{F}_{t}\right)_{t \in\left[t_{0}, t_{1}\right]}$ is called a martingale (with respect to the filtration $\mathcal{F}_{t}, t \in\left[t_{0}, t_{1}\right]$ ) if it satisfies the two following conditions:

1. $E\{|M(t)|\}<\infty, \quad \forall t$,

2. $E\left\{M(t) \mid \mathcal{F}_{s}\right\}=M(s), \quad t \geq s$.

The continuous martingale $X=\left(X(t), \mathcal{F}_{t}\right)_{t \in\left[t_{0}, t_{1}\right]}$ is called square-integrable if $E\left\{X(t)^{2}\right\}<\infty, \quad \forall t$ in $\left[t_{0}, t_{1}\right]$. Let $X=\left(X(t), \mathcal{F}_{t}\right)_{t \in\left[t_{0}, t_{1}\right]}$ be a continuous, square-integrable martingale with $X\left(t_{0}\right)=0$ with probability one. Then, 
the celebrated Doob-Meyer decomposition theorem implies that there exists a unique representation for $X(t)^{2}$

$$
X(t)^{2}=<X>(t)+M(t), \quad t \in\left[t_{0}, t_{1}\right]
$$

where $\langle X\rangle$ is an adapted, continuous, increasing process with $\langle X\rangle$ $\left(t_{0}\right)=0$, and $M$ is a continuous martingale with $M\left(t_{0}\right)=0$. The process $<X>(\cdot)$ is called the quadratic variation of $X(\cdot)$. Consider now two continuous, square-integrable martingales $X=\left(X(t), \mathcal{F}_{t}\right)_{t \in\left[t_{0}, t_{1}\right]}$ and $Y=$ $\left(Y(t), \mathcal{F}_{t}\right)_{t \in\left[t_{0}, t_{1}\right]}$. Their cross-variation process $\langle X, Y\rangle$ is defined for $t \in$ $\left[t_{0}, t_{1}\right]$ by

$$
<X, Y>(t):=\frac{1}{4}[<X+Y>(t)-<X-Y>(t)]
$$

The cross variation of $X$ and $Y$ is characterized by the fact that $X Y-<$ $X, Y>$ is a continuous martingale.

A nonnegative random variable $T(\omega)$ is called a Markov time or a stopping time (relative to the filtration $\mathcal{F}_{t}, t \in\left[t_{0}, t_{1}\right]$ ) if, for all $t$ in $\left[t_{0}, t_{1}\right]$,

$$
\{\omega: T(\omega) \leq t\} \in \mathcal{F}_{t}
$$

Let $T$ be a stopping time relative to $\mathcal{F}_{t}, t \in\left[t_{0}, t_{1}\right]$. Then, the $\sigma$-field $\mathcal{F}_{T}$ of events determined prior to the stopping time $T$ consists of those events $A \in \mathcal{A}$ for which $[A \cap\{\omega: T(\omega) \leq t\}] \in \mathcal{F}_{t}$, for every $t$ in $\left[t_{0}, t_{1}\right]$.

The stochastic process $M=\left(M(t), \mathcal{F}_{t}\right)_{t \geq 0}$, is called a local martingale if there exists an increasing sequence of stopping times (with respect to $\mathcal{F}$ ) $\left\{T_{n}\right\}, n=1,2, \ldots$ such that

1. $\mathbf{P}\left(\lim T_{n}=\infty\right)=1$; 
2. for any $n$, the "stopped process" $\left(M\left(t \wedge T_{n}\right), t \geq 0\right.$, is a martingale.

The decomposition result for the product of two square-integrable martingale extends to local martigales as follows. Let $X=\left(X(t), \mathcal{F}_{t}\right)_{t \in\left[t_{0}, t_{1}\right]}$ and $Y=$ $\left(Y(t), \mathcal{F}_{t}\right)_{t \in\left[t_{0}, t_{1}\right]}$ be two continuous, local martingales. Then there is a unique adapted process $\langle X, Y\rangle$ such that $X Y-\langle X, Y\rangle$ is a continuous local martingale. We write $\langle X\rangle$ instead of $\langle X, X\rangle$. We can now state $\mathrm{P}$. Levy's martingale characterization of the Wiener process.

Theorem A.1 Let $M=\left(M(t), \mathcal{F}_{t}\right)_{t \geq 0}$ be a continuous local martingale with $M(0)=0$ a.s. and quadratic variation $<M>(t)=t$. Then $M$ is a standard Wiener process.

As a corollary to this theorem, we have the following result showing that continuous local martingales can be viewed as time-changed Wiener processes.

Theorem A.2 Let $M=\left(M(t), \mathcal{F}_{t}\right)_{t \geq 0}$ be a continuous local martingale with $M(0)=0$ a.s. Suppose that $\lim _{t \rightarrow \infty}<M>(t)=\infty$ with probability one. Define, for $\tau \geq 0$, the stopping time $T(\tau):=\inf \{t \geq 0:<M>(t)>\tau\}$. Then the time-changed process $W=\left\{W(\tau):=M(T(\tau)), \mathcal{G}_{\tau}:=\mathcal{F}_{T(\tau)}\right)_{\tau \geq 0}$ is a standard Wiener process and we have, with probability one,

$$
M(t)=W(<M>(t)), \quad t \geq 0 .
$$

The multivariate extension of this theorem is due to F. B. Knight [15, p.179]. 
Theorem A.3 Let $\left.M=\left\{M(t)=\left(M^{1}(t), \ldots, M^{n}(t)\right), \mathcal{F}_{t}\right)_{t \geq 0}\right\}$ be a continuous process with $M(0)=0$ a.s. Suppose that the components $M^{i}$ are local martingales satisfying the two following conditions:

1. $\lim _{t \rightarrow \infty}<M^{i}>(t)=\infty, \quad i=1, \ldots, n$ a.s.;

2. $<M^{i}, M^{j}>(t)=0, \quad i \neq j, t \geq 0$.

Let $T_{i}(\tau):=\inf \left\{t \geq 0:<M^{i}>(t)>\tau\right\}, t \geq 0, i=1, \ldots, n$. Then the $W^{i}(\tau):=M^{i}\left(T_{i}(\tau)\right), \tau \geq 0, i=1, \ldots, n$ are independent, standard Wiener processes.

The nontrivial content of this theorem is that, although the $M^{i}$ are not independent, applying first the appropriate time-changes, and then forgetting the time changes, we get independent Wiener processes. Forgetting the timechanges consists in replacing the filtrations $\left\{\mathcal{G}_{\tau}^{i}\right\}$ with the poorer filtrations $\left\{\mathcal{F}_{\tau}^{W^{i}}\right\}$. 